\title{
Nudité et anthropophagie, tabous modernes revus par la Renaissance
}

\section{Vanessa Dias, Université de Waterloo}

Dans son discours sur l'altérité, Mathilde Schuhmacher constate que «c'est seulement après avoir fait l'expérience de l'Autre que l'on est capable de le comprendre et de l'accepter, de relativiser l'étrangeté de ses mœurs et partant, d'analyser les autres avec un regard critique » (43). Au seizième siècle, à la suite de la colonisation des Amériques par les Européens et durant une époque où les tensions religieuses s'intensifient en France ${ }^{1}$, les voyageurs de la Renaissance et les écrivains humanistes redoublent d'intérêt pour la traversée de l'océan et pour les peuples du Nouveau Monde. Leurs récits de voyage invitent alors le lecteur « à opérer un retour sur Soi grâce à un détour par l'Autre » (44). Nous allons étudier, dans le présent article, quatre relations de voyages: Les singularités de la France Antarctique (1557) d'André Thevet, L'histoire d'un voyage faict en la terre du Brésil (1578) de Jean de Léry, Le grand voyage du pays des Hurons (1632) de Gabriel Sagard et des extraits de la correspondance de Marie de l'Incarnation. Sous une lumière comparatiste, nous nous interrogerons à savoir si les écrits des explorateurs et des missionnaires de l'Ancien Régime ont influencé les relations entre Occidentaux et Autochtones au cours des siècles. Nous nous demanderons également si les tensions entre les cultures d'hier sont capables d'éclairer les conflits de nos jours. Ainsi, l'étude se divisera en deux parties qui nous permettront de disséquer les sujets tabous de la nudité et du cannibalisme afin de mieux comprendre comment ils sont traités. Nous traiterons premièrement la représentation de la nudité et de la sexualité dans chaque récit et deuxièmement, nous explorerons les descriptions de l'anthropophagie et de la torture. En gardant à l'esprit qu'à l'époque chacun de ces phénomènes était tout à fait singulier et difficile à accepter, nous déterminerons ensuite le rôle de la religion et des mœurs occidentales dans le refus ou la réflexion des auteurs, et nous examinerons dans quelle mesure les voyageurs se ressemblent ou s'opposent dans leurs narrations. Notre étude nous permettra de réfléchir sur la perspective des voyageurs et sur leur capacité (ou leur incapacité) à maintenir un regard critique sur le Nouveau Monde.

Le tabou de la nudité représente un premier concept problématique chez les Européens voyageant dans le Nouveau Monde. Nous entendons par le mot «tabou » le caractère interdit d'un lieu, d'un objet, d'une idée ou d'une personne désigné par la majorité ou plusieurs membres d'un 
groupe ou d'une communauté. Ainsi, le concept de «tabou»se rattache particulièrement à la religion et aux peuples à l'extérieur du royaume de France, ou les « sauvages » ${ }^{2}$. Bien que quelques auteurs apportent des réflexions importantes sur cette singularité et arrivent à la relativiser, d'autres la refusent totalement. En créant son premier portrait de l'Autochtone, Thevet se positionne du côté du refus et de la condamnation de la nudité : «[D]e gens merveilleusement étranges et sauvages, sans foi, sans loi, sans religion, sans civilité aucune, mais vivant comme bêtes irraisonnables [...], demeurant toujours nus tant hommes que femmes, jusques à tant, peut-être, qu'ils seront hantés des chrétiens, donc ils pourront peu à peu dépouiller cette brutalité pour vêtir une façon plus civile et humaine » (162). Tout d'abord, notons que le refus complet de la nudité se manifeste dans la métaphore suggestive à la fin de la citation alors que Thevet évoque l'habillement plus civilisé et humain que les Autochtones doivent revêtir. Cette image révèle clairement la position de Thevet. Sur le plan symbolique, Thevet fait référence à la Genèse et à Adam et Ève éprouvant la honte qui les amène à couvrir leur corps : «[N]ous devons louer affectueusement le Créateur qui nous a éclairci les choses, ne nous laissant ainsi brutaux comme ces pauvres Amériques » (162). Chez les chrétiens, le vêtement cache la nature corrompue de l'homme et, inversement, la nudité est le refus du premier péché. La femme est d'ailleurs souvent associée au mal dans l'œuvre de Thevet; c'est notamment à cause d'elle que les Autochtones (puis les Français) souffrent de la maladie des pians : «[C]e peuple est fort luxurieux, charnel et plus que brutal, les femmes spécialement, car elles cherchent et pratiquent tous les moyens à émouvoir les hommes au déduit »(236). Cette accusation renvoie à l'idée qu'Ève a condamné Adam pour l'éternité en le séduisant avec le fruit interdit. Thevet n'est capable ni de surmonter ni de mettre en perspective la nudité des Autochtones car elle ne reflète en rien le message tiré de la Bible, qui était la source de toutes les connaissances à l'époque.

La nudité est aussi problématique chez Léry, mais pour une autre raison. De religion réformée en lutte contre l'hypocrisie des catholiques, souvent représentée par les idées de Thevet, Léry hésite à condamner ou à approuver la nudité. Par conséquent, il prend des chemins détournés pour argumenter en faveur de la nudité sans faire l'éloge explicite de l'Autochtone. Le premier portrait que Léry peint en apercevant les Tupis (qui sont les Amérindiens de la côte brésilienne) fait ressortir une dissemblance par rapport au récit de Thevet : «[C]hose non moins estrange que difficile à croire à ceux qui ne l'ont veu, tant hommes, femmes qu'enfans, non seulement sans cacher aucunes parties de leurs corps, mais aussi sans monstrer aucun signe d'en avoir honte n'y 
vergogne, demeurent et vont coutumièrement aussi nuds qu'ils sortent du ventre de leurs mères » (212). Dans cet extrait, Léry constate que les Tupi n'éprouvent pas de honte face à leur nudité : le Tupi est ainsi un homme fier, tandis que 1'Autochtone chez Thevet est réduit à une bête irraisonnable. En dépit de son regard généralement extérieur et objectif, Léry refuse subtilement la nudité lorsqu'il nous offre le premier portrait de ses cinq contemplations du «sauvage » : «[I]maginez en vostre entendement un homme nud» (226), «[C]ouvrez-luy tout le corps [...] revetez-le » (227). Même si Léry ne refuse pas explicitement la nudité comme Thevet le fait, cette citation révèle un désir de la couvrir.

En Amérique du Nord, la nudité est moins fréquente chez les tribus autochtones de l'époque, mais elle est tout de même présente chez les Hurons/Wendats qui se débarrassent de leurs vêtements lors des cérémonies traditionnelles ou lors des danses rituelles. Bien que Sagard, frère récollet ${ }^{3}$, nous apporte un nombre important de renseignements sur une variété d'aspects de la vie des Wendats, il décrit très peu leur nudité. Il nous semble cependant que Sagard n'exprime pas un refus aussi extrême de la nudité que les autres voyageurs. Selon lui, la nudité des femmes indigènes ne les conduit pas à la luxure puisqu'elle permet à la chaleur du corps de s'échapper plus facilement et qu'elle réduit le désir sexuel : «[L]es garçons et jeunes hommes de Canada, et particulièrement du pays de nos Hurons, lesquels ont licence de s'adonner au mal sitôt qu'ils peuvent, et les jeunes filles de se prostituer sitôt qu'elles en sont capables, j'ose affirmer qu'ils sont moins sujets à ce vice que par-deçà, dont on peut attribuer la cause, partie à leur nudité [...] » (203). Nous pourrions attribuer sa perspective différente à plusieurs raisons, mais il semble bien que, par un raisonnement médical, il parvienne à donner une explication positive à la nudité, et à ainsi modifier la perception des Wendats que pourraient avoir les lecteurs. Dans l'édition critique de Réal Ouellet et Jack Warwick, on laisse d'ailleurs présumer que Sagard, dans ce genre de témoignage plus personnel, cherche à exprimer son admiration pour les nations autochtones, moins portées au péché que les Français.

En revanche, Marie de l'Incarnation, missionnaire catholique de l'ordre des Ursulines, fait constamment référence à la nudité à travers sa description des vêtements des jeunes filles de la Nouvelle-France. Pendant son travail d'évangélisation, elle cherche à imposer les valeurs européennes aux jeunes filles autochtones en leur enseignant à lire, en les habillant, et en leur apprenant à prier. À titre d'exemple, dans la lettre 43 de sa correspondance, Marie de l'Incarnation raconte une anecdote d'une jeune indigène appelée Marie Negabmat qui a refusé le monde des 
chrétiens : «[L]e R. Père le Jeune [...] envoya deux grandes filles Sauvages Chrétiennes qui demeurèrent quelque temps avec elle pour la fixer mais ce fut en vain, car elle s'enfuit quatre jours après dans les bois aiant mis en pièces une robe que nous luis avions donnée » (95). En gardant à l'esprit que la nudité, pour les chrétiens, est la manifestation d'une malpropreté morale et physique, il est évident que le fait de revêtir les jeunes filles de vêtements occidentaux représente une étape importante pour Marie de l'Incarnation vers la pureté des mœurs. Elle confirme cette volonté de purification par la suite : «[N]ous aimerions mieux manquer de tout, que de laisser nos filles dans la salleté insupportable qu'elles apportent de leurs cabanes. Quand on nous les donne elles sont nues comme un ver, et il les faut laver depuis la tête jusqu'aux pieds, à cause de la graisse dont leurs parens les oignent par tout le corps » (97). Dès lors, il s'agit symboliquement d'une véritable purification. On lui donne des vêtements, mais l'on doit, avant, lui enlever la graisse.

Il est certain que les auteurs de notre corpus condamnent tous la nudité : alors que Thevet et Marie de l'Incarnation la condamnent sans retour et en font un signe d'impureté, Léry et Sagard, un réformé et un frère récollet, ont des opinions moins tranchées. L'on pourrait évidemment se demander dans quelle mesure ces quatre auteurs saisissent la place de la nudité dans les imaginaires autochtones de l'époque. Rien n'est plus incertain, mais l'on peut tout de même espérer que Léry et Sagard ont, chacun à leur manière, permis une première compréhension de ce trait opposant les cultures autochtones américaines et européennes de la Renaissance et du dix-septième siècle.

Considérons, dans la deuxième partie de notre analyse, les représentations du cannibalisme, rituel autochtone qui suscite 1'horreur des Européens. Pour expliquer ce phénomène étrange, Thevet reste dans une position assez stricte et superficielle d'observateur. Cependant, il fait quelques constats qui semblent mal informés. Nous prenons, à titre d'exemple, le passage où Thevet explique la manière dont l'Autochtone désigné pour tuer le prisonnier que l'on s'apprête à manger se fera plusieurs incisions au corps : «Et la raison [...] est qu'il fait cela par plaisir, réputant à grande gloire ce meurtre par lui commis en la personne de son ennemi »(217). Si juste, en partie, que soit ce raisonnement, puisque les Autochtones font la guerre surtout pour se venger et pour faire peur à leurs ennemis, ramenant les prisonniers comme signe d'honneur et de gloire, Thevet n'arrive pas à expliquer la valeur purificatrice des incisions. De plus, il compare les incisions sur le corps aux règles des filles, en supposant que les filles font de telles incisions pendant trois jours après la «première purgation », et cela bien qu'il n'y ait pas de rapport entre ces deux rites. 
En revanche, Léry donne au lecteur une explication plus complète et nuancée que celle de Thevet. Il indique tout d'abord que les Tupi organisent des banquets anthropophages afin d'exprimer leur vengeance, pour faire peur à leurs ennemis. En outre, grâce à la mise en évidence de la vertu héroïque du prisonnier, le lecteur s'aperçoit à quel point le cannibalisme est une partie essentielle de la culture des Tupi : «Sur quoy pour monstrer qu'encores que ces nations barbares craignent fort la mort naturelle, neantmoins tels prisonniers s'estimans heureux de mourir ainsi publiquement au milieu de leurs ennemis » (358). Cette citation nous fait comprendre que le meurtre du prisonnier et le banquet qui suit, sont une coutume si inévitable et obligatoire que même les prisonniers sont à l'aise avec leur mort imminente. Cependant, le cannibalisme est condamné sans appel par Léry. Sa description des pratiques cannibales suscite l'horreur chez le lecteur car il la décrit en faisant allusion à la boucherie des animaux (360; 362-363), en décrivant le rituel du sang et en révélant avec dégoût que quelques truchements de Normandie (370) ont partagé le festin avec les indigènes. Malgré tout, et contrairement à Thevet, Léry parvient à relativiser le cannibalisme en rappelant qu'il y a dans la France catholique des personnes encore plus cruelles que les cannibales brésiliens : « [S]i on considere à bon escient ce que font nos gros usuriers (sucçans le sang et la moëlle, et par consequent mangeans tous en vie, tant de vefves, orphelins et autres pauvres personnes auxquels il vaudroit mieux couper la gorge tout d'un coup, que de les faire ainsi languir) qu'on dira qu'ils sont encores plus cruels que les sauvages dont je parle » (375).

Léry rappelle également le massacre de la Saint-Barthélemy, où des milliers de protestants ont été tués sans raison et jetés dans la rivière. Il n'essaie pas de montrer que le cannibalisme est moins condamnable, mais que les Européens ne peuvent pas s'arroger le droit de le condamner. Léry à la fois repousse et attire alors vers lui le monde Tupi : s'il ne participe pas aux banquets cannibales, il ne comprend pas non plus pourquoi les catholiques, qui massacrent d'autres chrétiens en faisant preuve d'une grande cruauté, condamnent les Tupis qui, après tout, tuent selon un rite guerrier faisant une grande place au courage et à la rapidité d'exécution. Comme le dit Montaigne plus tard, l'on pourrait se demander qui sont les êtres les plus humains : les Européens qui infligent souffrances, tortures et actes de barbarie aux vivants, ou les Brésiliens qui s'acharnent sur les morts.

Parallèlement à sa description de la nudité, Sagard aborde la notion de l'anthropophagie avec prudence. Nous n'avons trouvé que deux allusions à cette coutume dans des descriptions de festins des indigènes et de torture des ennemis : « $[\mathrm{P}]$ endant le festin, et après avoir vidé les 
chaudières et les compliments et remerciements rendus, ils partirent [...] pour entrer ès terres ennemies, sur lesquelles ils prirent environ soixante de leurs ennemis, la plupart desquels furent tués sur les lieux et les autres amenés en vie et faits mourir aux Hurons, puis mangés en festin » (238). Contrairement aux voyageurs que nous venons de citer, Sagard évacue rapidement le sujet. Il y reviendra une dernière fois à la fin de son ouvrage, après une longue description détaillée de la torture que les Autochtones ont fait subir à leurs ennemis :

Arrivés que sont les prisonniers en leur ville ou village, ils leur font endurer plusieurs et divers tourments. [...] ils leur arrachent les ongles et leur coupent trois principaux doigts. [...] ils leur lèvent toute la peau de la tête avec la chevelure et après ils y mettent du feu et des cendres chaudes [...], on lui coupe la tête ; puis on lui ouvre le ventre [...]. Le corps ainsi éventre et accommode, on le fait cuire dans une grande chaudière, puis on le mange en festin, avec liesse et réjouissance. (249)

La description de la torture est réalisée de façon élaborée, voire minutieuse, mais l'allusion à l'anthropophagie est minimaliste. Sagard essaie de dédramatiser le fait que ces prisonniers sont mangés lors d'un festin en faisant précéder ce constat par une description gênante de la torture et en déclarant que les Iroquois ou d'autres tribus font subir un sort encore pire à leurs prisonniers : «Quand les Iroquois ou autres ennemis peuvent attraper de nos gens, ils leur en font de même, et c'est à qui fera du pis à son ennemi » (249). Il paraît que le malaise est ainsi apaisé par une réflexion qui tente de tempérer, vis-à-vis d'autres groupes autochtones, la cruauté des Hurons de Sagard.

Outre la description de Sagard, Marie de l'Incarnation donne également beaucoup de place à son discours de la torture chez les Autochtones de l'Amérique de Nord. Dans la Lettre 110, elle fournit toute une description de la torture horrifique que les Iroquois faisaient subir aux chrétiens et ajoute une référence à l'anthropophagie avec une simple proposition en fin de phrase, un peu à la façon de Sagard : «[L]es hommes et les jeunes gens, qui étoient capables de porter les armes, furent distribuez en tous les Villages pour y être brûlez, bouillis et rôtis », ou encore « [Ils] jettèrent son corps dans le feu, ou il fut consumé » (327). Si Marie de l'Incarnation refuse de décrire l'anthropophagie dans ses lettres, cela n'empêche pas qu'elle relate l'histoire de la rupture de la paix par les Iroquois qui ont crucifié un enfant de trois ans :

Nous avons appris que ces Barbares plus cruels que les bêtes féroces crucifièrent avant que de partir de ce lieu un petit enfant âgé seulement de trois ans qui avoit été baptisé. Ils lui étendirent le corps sur une grosse écorce, et lui percèrent les pieds et les mains avec des bâtons pointus en forme de cloux. O que cet enfant est heureux d'avoir mérité en son état d'innocence l'honneur de mourir d'une mort semblable à celle de Jésus-Christ. (326) 
Il ne s'agit pas ici de cannibalisme, bien entendu, mais est-ce que Marie de l'Incarnation aurait pu comparer cet acte atroce au festin des Iroquois comme Léry a comparé les massacres perpétrés lors des guerres de religion en France, aux festins cannibales des Tupi ? La torture endurée par les chrétiens est si affreuse et barbare qu'elle est alors perçue comme un acte de bravoure et de martyre. Dans les deux cas, ce sont ainsi leurs coreligionnaires, les réformés pour Léry, les catholiques missionnaires pour Marie de l'Incarnation, qui sont les perdants ; leurs actions ne sont pas considérées comme plus morales ou plus acceptables par ces deux voyageurs. Les Tupis et les Hurons/Wendats s'élèvent du même coût dans l'échelle de l'humanité et de la rédemption.

Les représentations du cannibalisme et celles de la nudité, dans les quatre récits de voyage que nous avons analysés, laissent présager qu'un certain dialogue des altérités a pu se produire à l'époque des premières rencontres entre les Français et les tribus indigènes d'Amérique, même s'il fut bien timide. Les représentations en miroir, prenant comme point de référence des situations immorales remarquées en France, ont permis, de façon paradoxale, d'ouvrir le dialogue à l'altérité. Sagard, mais surtout Léry sont ainsi arrivés à relativiser des sujets tabous en s'élevant contre des pratiques plus barbares ou luxurieuses dont ils ont été témoins en France. L'on pourrait d'ailleurs se demander jusqu'où ce courant de sympathie et d'identification aux nations autochtones, que l'on retrouve d'ailleurs chez Montaigne, aurait pu aller si Léry et Sagard avaient pu prolonger leurs séjours en Amérique. Il est peut-être rassurant de penser qu'un certain dialogue, même éphémère, a donc pu avoir lieu avant que la conquête de l'Amérique ne fasse place à l'intolérance. Sagard, que l'on redécouvre dans les milieux de la recherche, pourrait peut-être aussi s'inscrire dans un même mouvement d'écoute, cette fois-ci en pleine Réforme tridentine ${ }^{4}$, d'une altérité prémoderne.

Relire des textes du passé n'est donc pas inutile pour comprendre notre époque. Qui sait, un Léry ou un Sagard, mais également une Marie de L'Incarnation et un Thevet, nous permettront peut-être, durant la réconciliation d'aujourd'hui, d'examiner le parcours des hommes et des femmes qui sont les premiers entrés en contact avec la richesse des cultures autochtones d'Amérique. D'une part, cette réflexion pourrait nous permettre de voir plus clairement la manière dont on confronte les différences de l'Autre. Les intolérances que l'on constate souvent dans les récits de voyage de la Renaissance se manifestent de nos jours devant d'autres diversités : origines ethniques, orientations sexuelles, croyances religieuses, alignements politiques, etc. D'autre part, les premiers dialogues (ou non-dialogues) entre Européens et Autochtones donnent le ton de ce rapport pour les siècles à venir. Dès lors, on se demande si les Européens auraient fait un effort 
pour comprendre l'Autre et non seulement le conquérir, si cela aurait changé la nature des relations futures qui resteront tendues et difficiles.

\section{Ouvrages cités}

La Bible de Jérusalem. Paris : Desclee de Brouwer, 1975.

Léry, Jean de. Histoire d'un voyage faict en la terre du Brésil. Paris : Poche, 1994 [1578].

Marie de l'Incarnation. Correspondances. Solesmes : Abbaye Saint-Pierre de Solesmes, 1971.

Ouellet, Réal. La relation de voyage en Amérique (XVI -XVIII ${ }^{e}$ siècles) : au carrefour des genres. Québec : PU Laval, 2010.

Sagard, Gabriel. Le grand voyage du pays des Hurons. Montréal : Bibliothèque québécoise, 2007 [1632].

Schuhmacher, Mathilde, dir. De l'un à l'autre : les discours sur l'altérité, de Montaigne à Grand Corps Malade. Paris : Flammarion, 2016.

Thevet, André. Les singularités de la France Antarctique. Paris : Chandeigne, 2011 [1557].

\footnotetext{
Notes

${ }^{1}$ À partir du seizième siècle, le protestantisme et le catholicisme s'opposent, incitant une série de conflits (les guerres de Religion) qui dévastent le royaume de France. Durant ce temps, de nombreux protestants et catholiques fuient la France, se réfugiant dans d'autres pays européens ou traversant l'océan pour découvrir le Nouveau Monde.

${ }^{2}$ Au seizième siècle, le terme « sauvage » n'avait pas la même connotation que de nos jours. À l'époque, «sauvage » se dit « de certains peuples qui vivent ordinairement dans les bois, presque sans religion, sans loix, sans habitation fixe \& plustost en bestes qu'en hommes. » (Dictionnaire de l'Académie française, 1694)

${ }^{3}$ Gabriel Sagard est un religieux réformé dans l'ordre de Saint-François. À l'époque, les autorités françaises «penchent pour une politique de pénétration continentale » (Sagard 12). Ainsi, les missionnaires se sont mis à traverser l'océan pour créer les premiers contacts avec les populations autochtones, pour apprendre leurs langues et leurs coutumes, et aussi pour « porter l'Évangile » (77).

${ }^{4} \mathrm{La}$ « Réforme tridentine » renvoie aussi à la réforme catholique ou à la Contre-Réforme. L'adjectif «tridentin » est issu du concile de Trente convoqué par Paul III en 1542.
} 